\title{
Progress and Prospects of Dog-Mediated Rabies Elimination in China
}

\author{
Wenwu Yin ${ }^{1, *}$; Zhen F. Fu; George F. Gao ${ }^{3}$
}

\section{CURRENT STATUS OF RABIES IN CHINA}

Through concerted stakeholder effort, rabies prevention and control in China has achieved remarkable results. The reported incidence of human rabies continues to decline at a rate of approximately $20 \%$ each year. In 2020, a total of 202 human rabies cases were reported countrywide, a decrease of $94 \%$ when compared with the most recent peak in 2007 (3,300 human cases). Likewise, rabies was reported only in 143 counties and districts, a decrease of $86 \%$ when compared to 984 counties and districts in 2007. More than 20 provincial-level administration divisions (PLADs) reported a drop in the number of cases to zero or single digits, demonstrating a sporadic trend in rabies occurrence. China has more than 2,800 counties and districts, and dog-mediated rabies may have been eliminated in some areas, which is a solid foundation for achieving the goal of eliminating dog-mediated rabies nationwide $(1-3)$.

Human rabies cases in China present the characteristics of "Tri-more," i.e., 1) there are more cases in rural areas and peasants account for over $65 \%$ of the total number of cases; 2) the number of male cases approximately doubles that of female cases; and 3 ) most of the cases are observed in children under 15 and people over 50 years of age.

Dogs are the major source of human rabies in China (3), accounting for approximately $95 \%$ of all cases, followed by cats (approximately 5\% of all cases). Foxes, raccoon dogs, badgers, and wolves are the main rabies hosts of wild animals, but less than $1 \%$ of human rabies were transmitted by wild animals.

Despite remarkable achievements in the past decade, rabies remains a serious health threat in China. In recent years, the number of deaths from rabies ranked among the top five reported notable infectious diseases in China, posing a huge threat to people's lives. The number of people who have to seek post-exposure prophylaxis (PEP) due to animal injury has been estimated to be as high as $8-10$ million annually in
China. It is estimated that the cost of human rabies vaccination, immunoglobulin, and wound medical treatment may exceed 7 billion CNY per year. The rabies epidemic not only seriously threatens people's lives, but also poses a heavy economic burden and has serious psychological impacts.

\section{STRATEGIES AND MEASURES FOR RABIES CONTROL IN CHINA}

The Chinese government established a governmentled, multisectoral-coordinated, "whole-society participation" mechanism under the concept of One Health in order to combat rabies. This approach entailed the following: 1) prioritizing elimination of dog rabies, including dog population control, registration, vaccination, quarantine, and movement management as dog vaccination remains the most costeffective way to prevent human rabies; 2) ensuring the availability, accessibility, and quality of PEP services to establish a second line of defense against rabies, while providing pre-exposure immunizations to high-risk groups, such as veterinarians and laboratory staff; 3) integrating human and animal health surveillance systems and implementing a containment strategy based on a One Health approach, once any human or dog cases are reported; and 4) promoting the welfare of dogs through advocacy, risk communication, and social mobilization.

The strategies and measures for rabies control are detailed as follows:

Active advocacy for the elimination of dog-mediated human rabies by 2030 as recommended by World Health Organization (WHO), a goal that has been highly prioritized by the Chinese government. The National Health and Family Planning Commission (NHFPC) [(currently National Health Commission (NHC)], Ministry of Public Security (MoPS), Ministry of Agriculture (MOA) [(currently Ministry of Agriculture and Rural Affairs (MARA)], and State Food and Drug Administration (SFDA) jointly issued the Current Status of Rabies Control in China in 
September 2009 (4). This document further defines the responsibilities of multiple sectors, establishing a multisectoral rabies prevention and control strategy, promoting close cooperation between various sectors, and actively carrying out prevention and control programs. It also defines major responsibilities for local governments, such as establishing grassroots organizations and strengthening dog management in rural areas.

Formulation and implementation of rabies prevention and control programs in China. The General Office of the State Council issued the National Medium and Long-Term Animal Epidemic Prevention Planning (2012-2020) on May 25, 2012 (5), which listed rabies as a zoonosis with priority in prevention and control and set a goal of achieving the control standard by 2020 . To implement the national plan, relevant departments have implemented their own prevention and control programs.

- The MOA has strengthened the prevention and control programs for animal rabies. The MOA issued and implemented the National Animal Rabies Prevention and Control Plan (2017-2020) (NYF [2017] No. 18) to strengthen rabies surveillance and response as well as dog immunization on June 2, 2017 (6). The MOA conducted large-scale dog immunization campaigns based on the "red collar" project. The MOA strengthened the core role of veterinarians and their sectors in rabies prevention and control and implemented compulsory rabies immunization regulations countrywide. The MOA further strengthened the countrywide dog surveillance and immunization system, especially in rural areas, to ensure the effective implementation of dog immunization measures in both rural and urban areas. As a result, the dog immunization rate has improved dramatically and the incidence of rabies in dogs has decreased, both of which led to the minimization of large-scale dog culling.

- Since 2005, the NHFPC has continued to implement the Central Government Transfer Payment National Rabies Surveillance Program in order to effectively conduct rabies surveillance, strengthen the epidemiological investigation of human rabies cases, and to improve multisectoral cooperation such as the sharing of surveillance information and promoting rabies responses. The NHFPC issued the Rabies PostExposure Prophylaxis Regulation in 2006 ( 7 ) and Technical Guidelines for Human Rabies Prevention and Control in 2016 (8) to standardize rabies PEP. Specifications for the Treatment Clinic Setting of Rabies Post-exposure Prophylaxis (T/CADERM
3010-2019) (9) were issued by Chinese Medical Rescue Association to strengthen the disposal clinic management of rabies PEP. Public health sectors have continuously strengthened training and conducted widespread professional rabies PEP training for grassroots outpatient doctors in various forms, including annual rabies meetings, online training, and standardized training courses for outpatient doctors at local injury clinics, and rabies awareness programs through official accounts and doctor groups on WeChat. Furthermore, the NHFPC promoted the incorporation of rabies vaccination and immunization into medical insurance in PLADs with high incidence of rabies to improve the accessibility and affordability of PEP services.

- The MoPS has continued to improve the registration and management of urban dogs.

- The SFDA has continued to strengthen the supervision of the quality and circulation of the human rabies vaccines and rabies immunoglobulins in order to supply high quality human rabies vaccines, and the supply of animal rabies vaccines is now sufficient to ensure the needs of prevention and control programs.

Strengthening rabies surveillance and utilizing integrated surveillance information. Based on case reports and post-exposure monitoring, surveillance of situations in which one dog bites multiple people has been emphasized in recent years. China CDC holds rabies laboratory testing training workshops every year to strengthen the construction of laboratory networks. Laboratory testing showed that the virus positive rate of one dog biting multiple people exceeded $80 \%$. The strengthening of epidemiological investigation, with the aim at achieving a case investigation rate of $100 \%$ as well as increasing investigation and responses, has been conducted.

Implementing surveillance, containment, and epidemic point and source elimination strategies. Rabies is a reemerging disease or imported infectious disease in most areas, and emergency response strategies should be implemented. All localities are supposed to strengthen joint prevention and control mechanisms and closely monitor and strengthen information sharing. Once a human or animal epidemic or "One dog biting multiple people" scenario emerges, a report must be submitted promptly to initiate the emergency response. Under the guidance of the government and multiple sectors including agricultural, health, and public security, among others, should cooperate closely, strengthen the implementation of various prevention and control measures in epidemic areas, and ensure the elimination 
of hidden dangers of epidemic spread in accordance with Technical Specification for Prevention and Control of Rabies. This includes the identification of epidemic areas and threatened areas, the culling of infected animals and other animals bitten by infected animals, the isolation of stray dogs, an emergency rabies immunization of all dogs and cats and restriction of their movement, and risk communication with the public.

Promoting the introduction of national laws and regulations related to rabies prevention and control as well as managing dogs according to law. Before 2021, there were no national, unified dog management regulations; moreover, local dog management regulations were only instituted in some regions. The Animal Epidemic Prevention Law of the People's Republic of China was revised and implemented on May 1, 2021 (10). It defined the main responsibilities of local governments in dog management and stipulated that dog owners should register and vaccinate dogs against rabies. When walking their dogs out of their houses, dog owners should ensure that their dogs have tags and adhere to necessary measures, such as tying dog leashes in accordance with the related regulations to prevent the dog from hurting people and spreading rabies.

Wide publicity, mass mobilization, active advocacy for civilized dog breeding and consolidation of the popular support. Mass media can actively facilitate the publicity of rabies prevention and control knowledge, which would enhance the public understanding of public health. Some non-governmental organizations can participate in the publicity and shelter of stray dogs.

\section{DIFFICULTIES AND CHALLENGES}

Currently, management of dogs is not yet optimal, and the registration rate is low particularly in rural areas. It is estimated that there are approximately 80-100 million dogs in China. The scattered breeding of dogs and cats is common, and this results in increasing danger to animals and human due to the spreading of rabies among animals and resulting transmission to humans. The supervision of $\mathrm{dog}$ breeding in rural areas is not well organized. It is not easy to implement immunization measures for rabies in dogs. Moreover, shelter for stray dogs should be expanded (11-13).

- The rate of vaccination in dogs is low in rural areas, posing difficultly in achieving $70 \%$ coverage in order to contain rabies transmission.
- People's awareness of civilized dog breeding needs to be improved. There are many phenomena, such as scattered breeding, absent registration, absent immunization, and random abandonment. Public understanding and involvement are key.

- The rabies epidemic is relatively serious in poor, marginal areas.

- The surveillance capacity is insufficient. Surveillance data for livestock and wild animals are insufficient. Furthermore, the rate of laboratory diagnosis in the reported cases of human rabies is low.

- The population of wild carnivores has increased recently; the spatial distribution has been restored or expanded; and small carnivores, such as weasels and raccoon dogs, have been increasing in cities. Thus, the risk of wild animal rabies has become increasingly prominent. Field surveillance demonstrates that foxes have become the main source of rabies infection from wild animals in China.

\section{KEY SUGGESTIONS ON RABIES PREVENTION AND CONTROL IN THE NEXT STAGE}

WHO and the World Organisation for Animal Health (OIE) jointly held the International Conference on Rabies Elimination in Geneva on December 10 and 11, 2015, to adopt an action plan and program framework for eliminating dog-mediated human rabies worldwide by 2030 (14-15).

The rabies epidemic has been effectively controlled in China but yet far away from elimination. China has complete public health and veterinary service systems, possesses the technologies for rabies prevention and control, and potentially produces and supplies animal and human rabies vaccines that meet international standards. China revised the Animal Epidemic Prevention Law of the People's Republic of China in 2021, which stipulates the requirement for dog owners to vaccinate dogs against rabies and perform civilized dog breeding. This law provides a national legal guarantee for the elimination of dog-mediated human rabies in China. Therefore, it is completely feasible for China to achieve the goal of "dog-mediated human rabies" in 2030, as proposed by the WHO. China should actively respond to the international call and action plan and commit to eliminating dog-mediated human rabies by 2030 .

Rabies elimination has entered the final stage. We should strive to eliminate dog-mediated human rabies and contribute to the health of China. China should 
continuously improve rabies surveillance, containment, and epidemic point and source elimination strategies as well as continue to exercise prevention and control measures, such as dog immunization and PEP.

Further attention is required. China should make an overall plan to eliminate rabies countrywide, increase investment, and strengthen the surveillance system. China should also strengthen animal rabies surveillance, including that of wild animals, improve the rate of laboratory diagnosis in reported cases, and continue to conduct surveillance data analysis and risk assessment of rabies.

Animal rabies prevention and control should be prioritized. China should continue routine dog management and immunization. The surveillance of rabies in wild animals is of paramount importance. In surrounding areas with wild-animal rabies foci, an immune barrier should be established to prevent the spread and spillover of wild-animal rabies to the dog population. Rabies risk in various places should be evaluated, with emphasis on the fact that the rate of dog immunization in high-risk areas must exceed $70 \%$.

PEP services should be provided continuously and service quality should be improved. China should update PEP specification, publicize, and implement PEP outpatient specifications, and introduce "integrated bite case management" with veterinary cooperation to ensure a more targeted use of PEP on the basis of risk assessments and diagnostic input and subsequently reduce administration of PEP for low-risk exposure.

The control of infectious sources should be increasingly emphasized and a rabies epidemic preparedness plan should be prepared. Various prevention and control measures, including the coordination of sector responsibilities; detection of rabies epidemics; initiation of the joint prevention and control mechanism; and cooperation with public security, agriculture, and other sectors, should be taken to eliminate the epidemic.

The Animal Epidemic Prevention Law of the People's Republic of China should be emphasized, civilized dog breeding should be promoted in accordance with the law, and the stray dog shelter system should be improved.

Multisectoral cooperation by NHC, MARA and other departments should be promoted in the certification of dog-mediated human rabies elimination in domestic areas and preparation for the countrywide elimination of dog-mediated human rabies.

Conflicts of interest: No conflicts of interest. doi: $10.46234 / \mathrm{ccdcw} 2021.205$

\#Corresponding author: Wenwu Yin, yinww@chinacdc.cn.

${ }^{1}$ Division of Infectious Disease, Chinese Center for Disease Control
and Prevention, Beijing, China; ${ }^{2}$ Department of Pathology, College of
Veterinary Medicine, University of Georgia, Athens, GA 30602; State
Key Laboratory of Agricultural Microbiology, College of Veterinary
Medicine, Huazhong Agricultural University, Wuhan, Hubei, China; ${ }^{3}$
Chinese Center for Disease Control and Prevention, Beijing, China.

Submitted: September 13, 2021; Accepted: September 22, 2021

\section{REFERENCES}

1. Tao XY, Liu SQ, Zhu WY, Rayner S. Rabies surveillance and control in China over the last twenty years. Biosaf Health 2021;3(3):142 - 7 . http://dx.doi.org/10.1016/j.bsheal.2020.11.004.

2. Chen QL, Ma XY, Rainey JJ, Li Y, Mu D, Tao XY, et al. Findings from the initial stepwise approach to rabies elimination (SARE) assessment in China, 2019. PLoS Negl Trop Dis 2021;15(3):e0009274. http://dx. doi.org/10.1371/journal.pntd.0009274.

3. Zhou H, Vong S, Liu K, Li Y, Mu D, Wang LP, et al. Human rabies in China, 1960-2014: a descriptive epidemiological study. PLoS Negl Trop Dis 2016;10(8):e0004874. http://dx.doi.org/10.1371/journal. pntd.0004874.

4. Chinese Ministry of Health, Ministry of Public Security, Chinese Ministry of Agriculture, Chinese Food and Drug Administration. Current status of rabies control in China. 2009. http://www.gov. cn/gzdt/2009-09/27/content_1428014.htm. (In Chinese).

5. National medium and long-term animal epidemic prevention planning (2012-2020). http://www.moa.gov.cn/zwllm/ghjh/201205/t20120530_ 2678977.htm. [2021-8-6]. (In Chinese).

6. Ministry of Agriculture and Rural Affairs of the People's Republic of China. National animal rabies prevention and control plan (2017-2020). 2017. http://www.moa.gov.cn/nybgb/2017/dlq/201712/ t20171231_6133713.htm. [2020-8-5]. (In Chinese).

7. Rabies post-exposure prophylaxis regulation. 2006. http://www.caaa.cn/ show/standard.php?ID=279. [2021-8-5]. (In Chinese).

8. Rabies post-exposure prophylaxis technical guidelines. 2016. https:// wenku.baidu.com/view/f55f342d30b765ce0508763231126edb6f1a76f 2.html. [2021-8-5]. (In Chinese).

9. China Medical Rescue Association. T/CADERM 3010-2019 Specifications for disposal clinic Setting of rabies exposure prophylaxisl. 2019. http://www.ttbz.org.cn/StandardManage/Detail/30007/. [20218-5]. (In Chinese).

10. The National People's Congress of the People's Republic of China. Animal epidemic prevention law of the People's Republic of China. 2021. http://www.npc.gov.cn/npc/c30834/202101/bcedb2c057984 cb680f8cc54529c0940.shtml. [2021-1-22]. (In Chinese).

11. Miao FM, Li N, Yang JJ, Chen T, Liu Y, Zhang SF, et al. Neglected challenges in the control of animal rabies in China. One Health 2021;12:100212. http://dx.doi.org/10.1016/j.onehlt.2021.100212.

12. Wang LH, Tang Q, Liang GD. Rabies and rabies virus in wildlife in mainland China, 1990-2013. Int J Infect Dis 2014;25:122-9. http://dx.doi.org/10.1016/j.ijid.2014.04.016.

13. World Health Organization. Global elimination of dog-mediated human rabies: report of the rabies global conference, Geneva, Switzerland, 10-11 December 2015. 2016. https://www.who.int/ publications/i/item/WHO-HTM-NTD-NZD-2016.02. [2020-8-5].

14. World Health Organization. Zero by 30: the global strategic plan to end human deaths from dog-mediated rabies by 2030. 2018. https:// www.who.int/publications/i/item/9789241513838. [2020-8-5].

15. Tu C, Feng Y, Wang Y. Animal rabies in the People's Republic of China. Rev Sci Tech 2018;37(2):519 - 28. http://dx.doi.org/10.20506/ rst.37.2.2820 